\title{
An Examination on Queuing Classification in Non-Destructive Testing
}

\author{
S. Radha, S. Maragathasundari, S.Sowmiyah, S. Kishore Eswar
}

\begin{abstract}
Nondestructive testing (NDT) is the way toward assessing, testing, or assessing materials, segments or gatherings for discontinuities, or contrasts in attributes without pulverizing the usefulness of the part or framework. Today current nondestructive tests are utilized in assembling, creation and in-administration investigations to guarantee item uprightness and unwavering quality, to control fabricating forms, lower generation costs and to keep up a uniform quality dimension. Amid development, NDT is utilized to guarantee the nature of materials and joining forms amid the manufacture and erection stages, and in-administration. NDT assessments are utilized to guarantee that the items being used keep on having the honesty important to guarantee their value and the security of the general population .Test strategy names regularly allude to the kind of entering medium or the hardware used to play out that test. One of the every now and again utilized test techniques is Liquid infiltrating test. The procedure is carried out in such a way that, to begin with, the penetrant is connected on the specimen which is to be tried, next the utilization of developer is done. In any case, before this designer procedure, there is a delay where other distractive procedure to be discounted. Next after the developer procedure, the procedure is rehashed if the aftereffect of the item isn't true to form. Following, maintenance work, for example, pre cleaning process like showering, brushing, inundating and so on is done. At last if in need another primer nondestructive test is additionally done for the item got. The above procedure is totally changed into a Queuing procedure in which the issue is all around concentrated by hypothesis of lining and it is illuminated by beneficial variable strategy. By the utilization of the above strategy, all the presentation proportions of the line in this procedure can be determined. Numerical delineation and graphical portrayal still amplify the model to a great extent.
\end{abstract}

Keywords: Delay, Compulsory vacation, stage 1, stage 2, optional stage 3 service

\section{INTRODUCTION}

Non Destructive Testing:

These destructive tests are regularly used to decide the physical properties of materials, for example, sway

Revised Manuscript Received on December 5, 2019

* Correspondence Author

S. Radha, Department of Mathematics, Kalasalingam Academy of Research and Education, Krishnankovil, Tamilnadu. Email: kradhai87@gmail.com

S. MaragathaSundari*, Department of Mathematics, Kalasalingam Academy of Research and Education, Krishnankovil, Tamilnadu. Email: maragatham01@gmail.com

S. Kishore Eswar, Department of Mechanical Engineering, Kalasalingam Academy of Research and Education, Krishnankovil, Tamilnadu. Email: kishoreeswar333@gmail.com

S. Sowmiyah, Department of Mathematics, Velammal Institute of Technology, Chennai, Tamilnadu. Email: sowmiyah.s@gmail.com obstruction, malleability, yield and extreme rigidity, break durability and exhaustion quality, however discontinuities and contrasts in material attributes are all the more viably found by [1] gave the condition appraisal for extension structure and it had been done utilizing diverse Non-Destructive strategies like Surface Ultrasonic Pulse Velocity Test, bounce back sledge , half - cell potential techniques and spread meter. "Reference [2] uncovered the split, broke and sound pieces of the steam turbine cutting edges which did not totally crack amid the investigation time frame". "Reference [3] Recognized and portrayed the most well-known fruitful strategies for NDT as connected to solid structures". "Reference [4] concentrated on structure steel structures utilized for plants, distribution centers and workplaces without flame security; the exhibition of steel auxiliary individuals for reuse is assessed by a Non-ruinous test". "Reference [5] had made the examination because of various elements (Characteristics of innovative phases of testing, unpleasantness of the tried surface and warm impacts) on the affectability and productivity of non-ruinous penetrant testing techniques". "Reference [11] talked about the standards of various non-damaging test methods and featured the use of these strategies for the assessment of castings". "Reference [12] surveyed the quality of a solid structure without making physical harm it, because of different reasons like its amazing significance or the legitimate contest on whether the quality of the solid in the structure was sufficiently tasteful or not. While trying to satisfy the need, relationship between's Destructive Test (DT) and Non-dangerous Test were done". "Reference [13] displayed the physical foundation of activity for radiography vortex present and ultrasonic assessment and examined their advantages, restrictions and run of the mill zones of uses". "Reference [14] exhibited a review on the guideline of activity for post-process assessment non-damaging testing methods". "Reference [15] introduced a methodology for looking at the nature of imperfection review materials dependent on a target quantitative premise (utilizing the perceivability of marker designs)". "Reference [6] examined the work on M/G/1 line with extra second stage administration and discretionary re service". "Reference [7] written about a solitary server line with two phase heterogeneous administration". "Reference [8] made an Analysis report on M/G/1 criticism line with three phases and numerous server excursions". "Reference [9] explored on a Non-Markovian Multistage Batch entry line with breakdown and reneging". 
"Reference [10] considered the Batch Arrival excursion line with second discretionary administration and Random Breakdowns". It ought to be noticed that while the medicinal field utilizes a large number of similar procedures, the expression "nondestructive testing" is commonly not used to depict therapeutic applications.

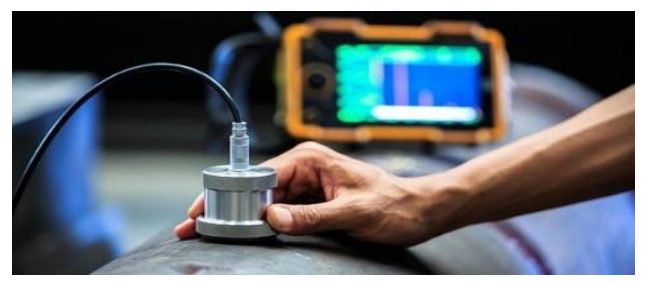

Fig.1

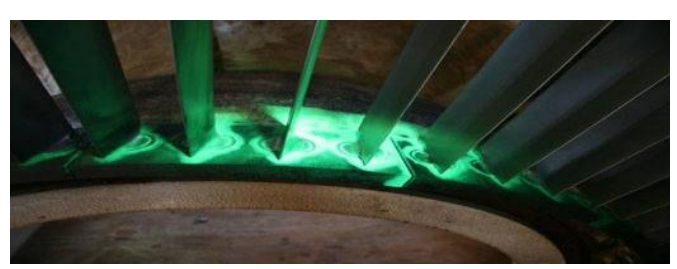

Fig.2

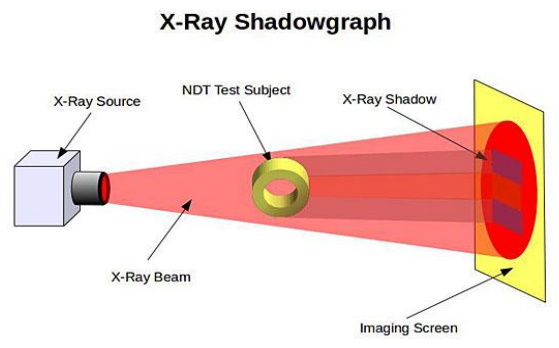

Fig.3

\section{NDT TEST METHODS}

The most frequently used test method is Liquid penetrating test. The method is explained in detail.

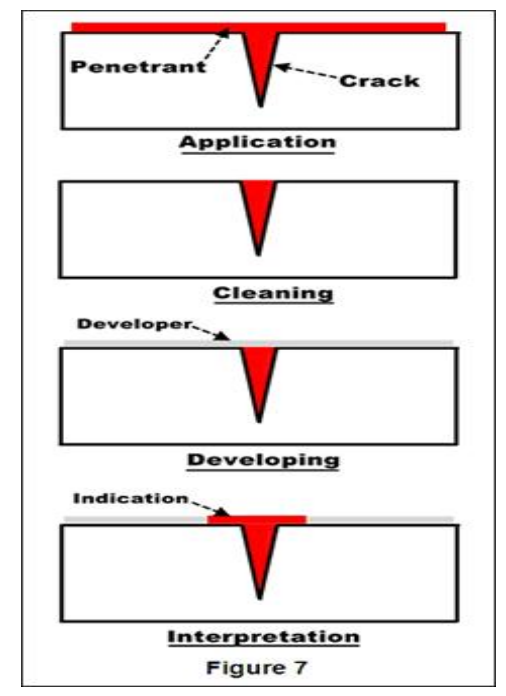

Fig.4 Liquid Penetrant Testing (PT)

The key standard of liquid penetrant testing is that when an outstandingly low thickness (fluid) liquid (the penetrant) is associated with the outside of an area, it will penetrate into holes and voids open to the surface. At the point when the excess penetrant is cleared, the penetrant got in those voids will stream retreat, making a sign. When playing out a PT survey, it is basic that the surface being attempted is immaculate and free of any outside materials resulting to applying the penetrant, it is permitted to sit externally for a foreordained time span by then the part is meticulously cleaned to oust plenitude penetrant from the surface. While expelling the penetrant, the official must be cautious so as not to clear any penetrant that has spilled into voids. A light covering of authority is then be related with the surface and given time to permit the penetrant from any voids or gap to spill up into the planner, making a perceivable sign. Following the bolstered maker stay time, the part is reviewed evidently, with the guide of a diminish light for fluorescent penetrants.

\section{PT TECHNIQUES}

\section{A. Solvent Removable}

Dissolvable removable penetrants require a dissolvable water to expel the overabundance penetrant. These penetrants are normally discernible in nature, for the most part tinted an amazing red shading that will separate well against a white organizer. The penetrant is normally sprinkled or brushed onto the part, by then after the penetrant stay time has finished, the part is cleaned with a surface hosed with penetrant cleaner after which the creator is related. Following the planner stay time, the part is evaluated to isolate any penetrant break out appearing through the specialist.

\section{B. Water Washable}

Water-launderable penetrants have an emulsifier joined into the penetrant that permits the penetrant to be removed utilizing a water sprinkle. They are a significant part of the time related by dunking the part in a penetrant tank, in any case the penetrant might be related with enormous parts by showering or brushing. Right when the part is completely confirmed with penetrant, the part is resolved to a channel burden up for the penetrant stay time, by then taken to a flush station where it is washed with a course water sprinkle to clear the wealth penetrant. Right when the wealth penetrant has been expelled, the part might be set in a warm air dryer or before a delicate fan until the water has been cleared. The part would then have the alternative to be set in a dry expert tank and verified with organizer.

\section{Post-emulsifiable}

Post-emulsifiable penetrants like lipophilic or hydrophilic are associated in a same way, anyway going before the water-washing step, emulsifier is associated with the surface for a prescribed time period (emulsifier withstand) to empty the excess penetrant. Exactly when the emulsifier withstand time has snuck past, the part is presented to a comparative water wash and making process used for water-launderable penetrants.

The over three sorts of penetrants are utilized in LPT or PT. This method experiences the accompanying stages: Phase1, Delay time, Phase 2, Feedback administration, excursion and Phase 3 (Optional) 


\section{1) Phase 1}

In stage one procedure we will apply the penetrant on the example to be tried. Penetrant that we will apply on the example will in two sorts. They are Fluorescent and Visible. Unmistakable penetrant is performed under white light while fluorescent penetrant must be performed utilizing a bright light in obscured territory. All will be all in the dimension 1 affectability range. Fluorescent penetrant is more delicate than noticeable penetrant in light of the fact that the eye is increasingly touchy to a splendid sign on a dim foundation. Affectability ranges from 1 to 4 . Penetrant are connected through water launderable strategy generally. Using slender components this procedure is connected.

\section{2) Delay time}

The penetrant that we apply on the example, won't settle on the example rapidly. Since in this strategy we are going to discover the breaks that are accessible on the example, the deformities on the example will fluctuate. Essentially in businesses, they complete four principle fabricating procedure with the end goal that moving, throwing, producing and welding. Moving deformities, for example, splits, creases, overlays, wavy. Giving imperfections such a role as virus closes, hot tears, porosity, blow openings, shrinkage. Forgings imperfections, for example, splits, laps, outer blasts. Welding, for example, breaks, porosity, undercut, cover, absence of combination, absence of combination, absence of entrance. Hence dependent on these assembling surrenders the penetrant that we apply on the example will set aside some effort to settle on them. There emerges the postpone time for this procedure. For minor size deformities, the defer time is short. For significant size deformities, the postpone time is huge. Since the thickness and consistency pursue of the penetrant will expends additional time.

3) Phase 2

After settlement of penetrant in the example, overabundance of penetrant will be expelled. Also, use of the designer is included; the job of engineer is to haul caught penetrant out of the imperfections and to spread it out superficially so it very well may be seen. Likewise gives light foundations to build differentiate when noticeable penetrant is utilized. Designer materials are accessible in a few distinct structures. They are dry powder, wet (water suspendable), wet (water dissolvable) and wet (non watery). The penetrant "enters" into surface breaking discontinuities by means of slender activity and different components. Along these lines, utilizing this instrument we will recognize the spot of deformities on the given example. Later on the investigation factor we address the imperfections.

4) Feedback service

In some cases appearance of the mistake may occur in stage 1 and stage 2, it might be either orderly blunder or irregular blunder. As these procedure are done physically blunder will show up. For the most part in these procedures we can discover about 50-90\%. Rules for right utilization of measurable technique to POD (Probability of discovery) tests can be found in ASTM E2862 standard practice for likelihood of recognition investigation for hit/miss information and MIL-HDBK-1823A non-dangerous assessment framework dependability evaluation from the U.S. division of safeguard handbook. Consequently, indeed similar stages are been rehashed.

\section{Vacation}

By applying distinctive sort of penetrant strategy, we can at present more increment the procedure progressively productive and quicker. We can apply post emulsifiable-(lipophilic). In organization or in ventures they call it as strategy B. we can likewise apply other two techniques like dissolvable removable and postemulsifiable-(hydrophilic) strategy. They are called as strategy $\mathrm{C}$ and $\mathrm{D}$ in enterprises. We can likewise pursue pre-cleaning process. Parts must be free of earth, rust, scale, oil, oil, and so forth to play out a dependable investigation. The cleaning procedure must expel contaminants from the surfaces of the part and deserts and should not plug any of the deformities. Pre-cleaning is the most significant advance in the PT procedure. There are numerous techniques for applications are conceivable, for example, brushing, showering, plunging/inundating, stream on and that's just the beginning.

\section{E. Stage 3 (discretionary)}

Once, the specimenis treated in non-dangerous strategies, the specimen is emitted to go about as an example (with the end goal that the up and coming items are been produce utilizing this as the parent or the copy of the article is finished). Else the example is permit to go in item stream chain. Now and again the example can likewise experience other non-damaging systems. Other non-ruinous strategies like Acoustic Emission testing (AET), Electromagnetic testing (ET), Radiographic testing (RT), Visual Inspection, and so on;

\section{MATHEMATICAL ASSUMPTIONS UNDERLYING THE MODEL}

Let $\xi_{1}(x)$ be the conditional probability of stage 1 service with distribution function $\bar{u}(\mathrm{x})$ and density function $g(x)$ and it is given by

$$
\xi_{1}(x)=\frac{u(x)}{1-\bar{u}(\mathrm{x})} \text { and } u(\mathrm{x})=\xi_{1}(x) \exp \left[-\int_{0}^{x} \xi_{1}(v) d v\right]
$$

Likewise, for the various parameters like delay, second stage, compulsory vacation and optional third stage of service respectively, we have

$$
\begin{array}{ll}
\xi_{2}(x)=\frac{v(x)}{1-\bar{v}(\mathrm{x})} \text { and } & v(\mathrm{x})=\xi_{2}(x) \exp \left[-\int_{0}^{x} \xi_{2}(v) d v\right] \\
\xi_{3}(x)=\frac{w(x)}{1-\bar{w}(x)} \text { and } & w(\mathrm{x})=\xi_{3}(x) \exp \left[-\int_{0}^{x} \xi_{3}(v) d v\right] \\
\xi_{4}(x)=\frac{g(x)}{1-\bar{x}(x)} \text { and } g(\mathrm{x})=\xi_{4}(x) \exp \left[-\int_{0}^{x} \xi_{4}(v) d v\right] \\
\xi_{5}(x)=\frac{y(x)}{1-\bar{y}(x)} \text { and } y(\mathrm{x})=\xi_{5}(x) \exp
\end{array}
$$

\section{GOVERNING EQUATIONS OF THE MODEL}

For the defined queuing model, the governing equations are as follows:

$$
\begin{aligned}
& \frac{d}{d x} B_{n}^{(1)}(x)+\left(\lambda+\xi_{1}(x)\right) B_{n}^{(1)}(x)=\lambda \sum_{j=1}^{n} d_{j} B_{n-j}^{(1)}(x) . \\
& \frac{d}{d x} B_{0}^{(1)}(x)+\left(\lambda+\xi_{1}(x)\right) B_{0}^{(1)}(x)=0 \text {. } \\
& \frac{d}{d x} T_{n}^{(d)}(x)+\left(\lambda+\xi_{2}(x)\right) T_{n}^{(d)}(x)=\lambda \sum_{j=1}^{n} d_{j} T_{n-j}^{(d)}(x) \\
& \frac{d}{d x} T_{0}^{(d)}(x)+\left(\lambda+\xi_{2}(x)\right) T_{0}^{(d)}(x)=0 \text {. }
\end{aligned}
$$

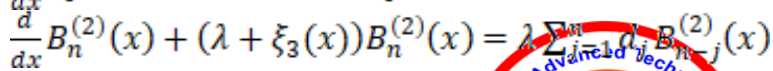


$\frac{d}{d x} B_{0}^{(2)}(x)+\left(\lambda+\xi_{3}(x)\right) B_{0}^{(2)}(x)=0$.

$\frac{d}{d x} V_{n}^{(c)}(x)+\left(\lambda+\xi_{4}(x)\right) V_{n}^{(c)}(x)=\lambda \sum_{j=1}^{n} d_{j} V_{n-j}^{(c)}(x)$

$\frac{d}{d x} V_{0}^{(c)}(x)+\left(\lambda+\xi_{4}(x)\right) V_{0}^{(c)}(x)=0$.

$\frac{d x}{d x} B_{n}^{(3)}(x)+\left(\lambda+\xi_{5}(x)\right) B_{n}^{(3)}(x)=\lambda \sum_{j=1}^{n} d_{j} B_{n-j}^{(3)}(x)$.

$\frac{d}{d x} B_{0}^{(3)}(x)+\left(\lambda+\xi_{5}(x)\right) B_{0}^{(3)}(x)=0$.

$\lambda Q=(1-r) \int_{0}^{\infty} V_{0}^{(c)}(x) \xi_{4}(x) d x+\int_{0}^{\infty} B_{0}^{(3)}(x) \xi_{5}(x) d x$

Boundary conditions:

The following boundary conditions are used to solve the above equations:

$B_{n}^{(1)}(0)=$

$(1-r) \int_{0}^{\infty} V_{n+1}^{(c)}(x) \xi_{4}(x) d x+p \int_{0}^{\infty} B_{n}^{(1)}(x) \xi_{1}(x) d x+$ $\int_{0}^{\infty} B_{n+1}^{(3)}(x) \xi_{5}(x) d x+\lambda D_{n+1} Q$

$T_{n}^{(d)}(0)=(1-p) \int_{0}^{\infty} B_{n}^{(1)}(x) \xi_{1}(x) d x$.

$B_{n}^{(2)}(0)=\int_{0}^{\infty} T_{n}^{(d)}(x) \xi_{2}(x) d x$.

$V_{n}^{(c)}(0)=\int_{0}^{\infty} B_{n}^{(2)}(x) \xi_{3}(x) d x$.

$B_{n}^{(3)}(0)=r \int_{0}^{\infty} V_{n}^{(c)}(x) \xi_{4}(x) d x$.

\section{DISTRIBUTION OF QUEUE LENGTH}

To solve (1) to (10), we follow the procedure given below:

We multiply (1) by $z^{n}$ and sum over ${ }^{n}$ from 1 to ${ }^{\infty}$ and add it to (2) we get,

$$
\frac{d}{d x} B^{(1)}(x, z)+\left(\lambda-\lambda D(z)+\xi_{1}(x)\right) B^{(1)}(x, z)=0
$$

Similarly,

$$
\begin{aligned}
& \frac{d}{d x} T^{(d)}(x, z)+\left(\lambda-\lambda D(z)+\xi_{2}(x)\right) T^{(d)}(x, z)=0 \\
& \frac{d}{d x} B^{(2)}(x, z)+\left(\lambda-\lambda D(z)+\xi_{3}(x)\right) B^{(2)}(x, z)=0 \\
& \frac{d}{d x} V^{(c)}(x, z)+\left(\lambda-\lambda D(z)+\xi_{4}(x)\right) V^{(c)}(x, z)=0 \\
& \frac{d}{d x} B^{(3)}(x, z)+\left(\lambda-\lambda D(z)+\xi_{5}(x)\right) B^{(3)}(x, z)=0 .
\end{aligned}
$$

Multiply (12) by $z^{n+1}$ and summing over ${ }^{n}$ from 0 to ${ }^{\infty}$, we get

$$
\begin{aligned}
& z B^{(1)}(0, z)=(1-r) \int_{0}^{\infty} V^{(c)}(x, z) \xi_{4}(x) d x- \\
& (1-r) \int_{0}^{\infty} V_{0}^{(c)}(x) \xi_{4}(x) d x+p \int_{0}^{\infty} B^{(1)}(x, z) \xi_{1}(x) d x- \\
& \int_{0}^{\infty} B^{(3)}(x, z) \xi_{5}(x) d x-\int_{0}^{\infty} B^{(3)}(x) \xi_{5}(x) d x+\lambda D(z) Q \\
& \text { Using }(11) \text { in above, we get } \\
& z B^{(1)}(0, z)=(1-r) \int_{0}^{\infty} V^{(c)}(x, z) \xi_{4}(x) d x+ \\
& p \int_{0}^{\infty} B^{(1)}(x, z) \xi_{1}(x) d x+\lambda(D(z)-1) Q
\end{aligned}
$$

Now integrating (17) from 0 to ${ }^{x}$ yields, $B^{(1)}(x, z)=B^{(1)}(0, z) e^{-(\lambda-\lambda D(z))-\int_{0}^{x} \xi_{1}(t) d t}$.

Integrating (23) by parts with respect to ${ }^{x}$ yields $B^{(1)}(z)=B^{(1)}(0, z) \frac{(1-\bar{u}(a))}{a}, a=\lambda-\lambda D(z)$.

Multiply both sides of (23) by $\xi_{1}(x)$ and integrating we get,

$$
\int_{0}^{\infty} B^{(1)}(x, z) \xi_{1}(x) d x=B^{(1)}(0, z) \bar{u}(a)
$$

Similarly,

$$
\begin{aligned}
& T^{(d)}(z)= \\
& T^{(d)}(0, z) \frac{(1-p(a))}{a}= \\
& (1- \\
& p) B^{(1)}(0, z) \bar{u}(a) \frac{(1-p(a))}{a} \\
& \int_{0}^{\infty} T^{(d)}(x, z) \xi_{2}(x) d x=(1-p) B^{(1)}(0, z) \bar{u}(a) \bar{v}(a) . \\
& \quad B^{(2)}(z)=B^{(2)}(0, z) \frac{(1-\bar{w}(a))}{a}= \\
& \quad(1-p) B^{(1)}(0, z) \bar{u}(a) \bar{v}(a) \frac{(1-\bar{w}(a))}{a}
\end{aligned}
$$

$\int_{0}^{\infty} B^{(2)}(x, z) \xi_{3}(x) d x=(1-p) B^{(1)}(0, z) \bar{u}(a) \bar{v}(a) \bar{w}(a)$

$$
\begin{aligned}
& V^{(c)}(z)=V^{(c)}(0, z) \frac{(1-\bar{x}(a))}{a}= \\
& (1-p) B^{(1)}(0, z) \bar{u}(a) \bar{v}(a) \bar{w}(a) \frac{(1-\bar{x}(a))}{a} \\
& \int_{0}^{\infty} V^{(c)}(x, z) \xi_{4}(x) d x= \\
& (1-p) B^{(1)}(0, z) \bar{u}(a) \bar{v}(a) \bar{w}(a) \bar{x}(a) \\
& B^{(3)}(z)=B^{(3)}(0, z) \frac{(1-\bar{y}(a))}{a}= \\
& r(1-p) B^{(1)}(0, z) \bar{u}(a) \bar{v}(a) \bar{w}(a) \bar{x}(a) \frac{(1-\bar{y}(a))}{a}
\end{aligned}
$$

$\int_{0}^{\infty} B^{(3)}(x, z) \xi_{5}(x) d x=$ $r(1-p) B^{(1)}(0, z) \bar{u}(a) \bar{v}(a) \bar{w}(a) \bar{x}(a) \bar{y}(a)$

Substituting (25) and (31) in (22), we get

$$
B^{(1)}(0, z)=\frac{Q \lambda(D(z)-1)}{z-(1-r)(1-p) \bar{u}(a) \bar{v}(a) \bar{w}(a) \bar{x}(a)-p \bar{u}(a)} \text {. }
$$

Hence equation (24),(26),(28),(30),(32) becomes,

$$
\begin{aligned}
B^{(1)}(z) & =\frac{-Q \lambda(1-\bar{u}(a))}{z-(1-r)(1-p) \bar{u}(a) \bar{v}(a) \bar{w}(a) \bar{x}(a)-p \bar{u}(a)} . \\
T^{(d)}(z) & =\frac{-Q \lambda(1-p) \bar{u}(a)(1-\bar{v}(a))}{z-(1-r)(1-p) \bar{u}(a) \bar{v}(a) \bar{w}(a) \bar{x}(a)-p \bar{u}(a)} .
\end{aligned}
$$$$
B^{(2)}(z)=\frac{-Q \lambda(1-p) \bar{u}(a) \bar{v}(a)(1-\bar{w}(a))}{z-(1-r)(1-p) \bar{u}(a) \bar{v}(a) \bar{w}(a) \bar{w}(a)-p \bar{u}(a)} .
$$

$V^{(c)}(z)=\frac{-Q \lambda(1-p) \bar{u}(a) \bar{v}(a) \bar{w}(a)(1-\bar{x}(a))}{z-(1-r)(1-p) \bar{u}(a) \bar{v}(a) \bar{w}(a) \bar{x}(a)-p \bar{u}(a)}$.

$B^{(3)}(z)=\frac{-Q \lambda r(1-p) \bar{u}(a) \bar{v}(a) \bar{w}(a) \bar{x}(a)(1-\bar{y}(a))}{z-(1-r)(1-p) \bar{u}(a) \bar{v}(a) \bar{w}(a) \bar{x}(a)-p \bar{u}(a)}$

\section{LIKELIHOOD CREATING CAPACITY OF THE LINE ESTIMATE}

Let $G^{*}(z)$ be the probability generating function of the queue length such that $G^{*}(z)=B^{(1)}(z)+T^{(d)}(z)+B^{(2)}(z)+V^{(c)}(z)+B^{(3)}(z)-S^{(t)}(z)$ Adding (35)-(39), we get

$$
G^{*}(z)=\frac{-Q \lambda\left[(1-\bar{u}(a))+(1-p) \bar{u}(a)\left\{\begin{array}{c}
(1-\bar{v}(a))+\bar{v}(a)(1-\bar{v}(a))+ \\
\bar{w}(a)(1-\bar{x}(a))+\bar{v}(a) \bar{w}(a) \bar{x}(a) \\
(1-\bar{y}(a)
\end{array}\right\}\right]}{z-(1-r)(1-p) \bar{u}(a) \bar{v}(a) \bar{w}(a) \bar{x}(a)-p \bar{u}(a)}
$$

The idle time $Q$ is determined by using the normalization condition

$$
G^{*}(1)+Q=1 \text {. }
$$


Using L'Hopital'srule, we get

$\lim _{z \rightarrow 1} G^{*}(z)=\frac{N^{\prime}(1)}{D^{\prime}(1)}$

Also $Q=\frac{D^{\prime}(1)}{D^{I}(1)+N^{\prime}(1)}$

From $Q$, the utilization factor $\rho$ can be determined.

\section{SYSTEM QUEUE EXECUTION PROCEDURES}

Let $L_{q}$ a chance to demonstrate the reliable state typical number of customers in the line. By then

$$
L_{q}=\left.\frac{d}{d z} G^{*}(z)\right|_{z=1}=\left.\frac{d}{d z}\left\{\frac{N(z)}{D(z)}\right\}\right|_{z=1}
$$

Where $N(z)$ and $D(Z)$ are the numerator and denominator of equation .

Since $G^{*}(z)=\frac{0}{0 a t}=1$, we utilize two fold separation and get

$$
L_{q}=\lim _{z \rightarrow 1} \frac{d}{d z} G^{*}(z)=\frac{D^{\prime}(1) N^{\prime \prime}(1)-D^{\prime \prime}(1) N^{\prime}(1)}{2\left(D^{\prime}(1)\right)^{2}} .
$$

Where primes mean subordinates with respect to $\mathrm{z}$ and after a course of action of logarithmic enhancement, we get length of the queue $L_{q}$ in closed frame.

$$
\begin{aligned}
& D^{\prime}(1)=\{1-(1-r)(1-p) \lambda[E(u)+E(v)+E(w)+ \\
& E(x)] p E(u) \lambda\}
\end{aligned}
$$$$
D^{n}=-(1-r)(1-p)\left[\lambda^{2} E\left(u^{2}\right)+\lambda^{2} E(u)[E(v)+E(w)+\right.
$$$$
E(x)]+\lambda^{2} E(v)[E(u)+E(x)+E(w)]+\lambda^{2} E(w)[E(u)+
$$$$
\left.E(v)+E(x)]+\lambda^{2} E(x)[E(u)+E(v)+E(w)]\right]-
$$$$
p \lambda^{2} E\left(u^{2}\right)
$$$$
N^{\prime}=-\lambda[-\lambda E(u)+(1-p)\{-\lambda E(w)-\lambda E(x)-
$$$$
\lambda r E(y)\}]
$$

$$
\begin{aligned}
& N^{n}=-\lambda\left[( 1 - p ) \left[-E(u) E(v) \lambda^{2}-\lambda^{2} E(u) E(w)+\right.\right. \\
& E\left(v^{2}\right) \lambda^{2}-\lambda^{2}(E(u)+E(v)) E(w)-E\left(w^{2}\right) \lambda^{2}- \\
& \lambda^{2} E(u) E(x)-\lambda^{2} E(v) E(x)-\lambda^{2} E(w) E(x)- \\
& \lambda^{2}(E(u)+E(v)+E(w)) E(x)-\lambda^{2} E\left(x^{2}\right)- \\
& r \lambda^{2} E(y) E(u)-r \lambda^{2} E(y) E(v)-r \lambda^{2} E(y) E(w)- \\
& r \lambda^{2} E(y) E(x)-\lambda^{2}(E(u)+E(v)+E(w)+E(x)) E(y)- \\
& \left.\left.\lambda^{2} E\left(y^{2}\right)\right]\right]
\end{aligned}
$$

Substituting for $N^{\prime}(1), N^{\prime \prime}(1), D^{\prime}(1), D^{\prime \prime}(1)$ from (42)(45) in (41), we obtain $L_{q}$ in closed form.

Further the mean waiting time of a customer in the queue as well as in the system and the number of customers waiting in the system can be found using Little's formula

$$
W_{q}=\frac{L_{q}}{\lambda}, W=\frac{L}{\lambda}, L=L_{q}+\rho .
$$

\section{NUMERICAL ILLUSTRATION}

Assume that service time follows exponential distribution in particular and based on this condition, the numerical justification is elaborated below. The values are collected accordingly:

$$
\begin{aligned}
& \xi_{1}=2, \xi_{2}=3, \xi_{3}=2.5, \xi_{4}=3.5, \xi_{5}=4, \lambda=3, E(u)= \\
& \frac{1}{\xi_{1}}, E(v)=\frac{1}{\xi_{2}}, E(w)=\frac{1}{\xi_{3}}, E(x)=\frac{1}{\xi_{4}}, E(y)=\frac{1}{\xi_{5}}, E\left(u^{2}\right)= \\
& \frac{2}{\xi_{1}{ }^{2}}, E\left(v^{2}\right)=\frac{2}{\xi_{2}{ }^{2}}, E\left(w^{2}\right)=\frac{2}{\xi_{3}{ }^{2}}, E\left(x^{2}\right)=\frac{2}{\xi_{4}{ }^{2}}, E\left(y^{2}\right)=\frac{2}{\xi_{5}{ }^{2}}
\end{aligned}
$$

Table I

Effect of change of $r=0.6,0.8,1,1.2,1.4$

\begin{tabular}{|l|l|l|l|l|l|}
\hline $\mathrm{Q}$ & $\rho$ & $L_{q}$ & $L$ & $W_{q}$ & $W$ \\
\hline 0.1775 & 0.8225 & 5.0236 & 5.8461 & 1.6745 & 1.9487 \\
\hline 0.1553 & 0.8447 & 8.0575 & 8.9022 & 2.6858 & 2.9674 \\
\hline 0.1306 & 0.8694 & 13.8706 & 14.7400 & 4.6235 & 4.9133 \\
\hline 0.1030 & 0.8970 & 27.0147 & 27.9117 & 9.0049 & 9.3039 \\
\hline 0.0720 & 0.9280 & 66.8416 & 67.7696 & 22.2805 & 22.5899 \\
\hline
\end{tabular}

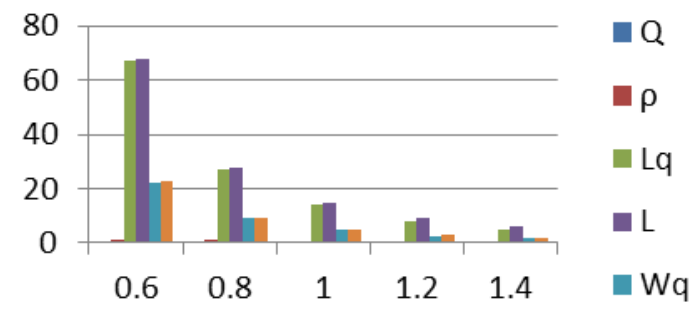

Fig. 5 Variation of $p$

Table II

Effect of change of $P=0.7,0.8,0.9,1,1.1$

\begin{tabular}{|l|l|l|l|l|l|}
\hline $\mathrm{Q}$ & $\rho$ & $L_{q}$ & $L$ & $W_{\text {q }}$ & $W$ \\
\hline 0.0593 & 0.9407 & 125.9009 & 126.8416 & 41.9670 & 42.281 \\
\hline 0.0857 & 0.9143 & 68.2767 & 69.1910 & 22.7589 & 23.064 \\
\hline 0.1255 & 0.8745 & 26.6803 & 27.5548 & 8.8934 & 9.1849 \\
\hline 0.1818 & 0.8182 & 10.1250 & 10.9432 & 3.3750 & 3.6477 \\
\hline 0.2577 & 0.7423 & 1.6710 & 2.4133 & 0.5570 & 0.8044 \\
\hline
\end{tabular}

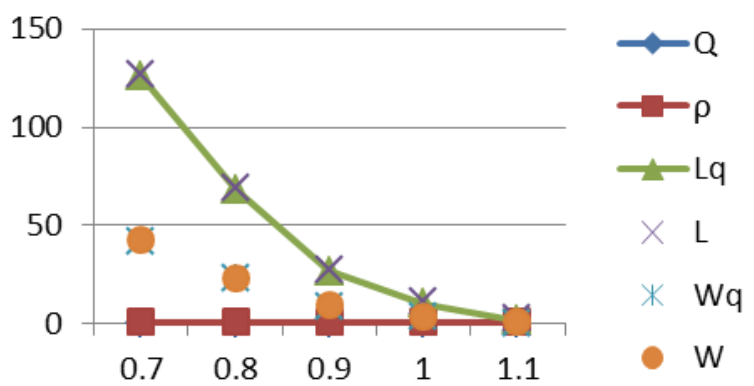

Fig. 6 Variation of $p$

\section{NUMERICAL REPORT}

From table 1 it is clear that if the option of taking stage 3 service increases, the idle time of the server for this particular stage gets decreases. Hence the other performance measures get amplified. From table 2, the results are as expected, that is if the probability of getting into feedback service increases, it leads to an increase in all the queue performance measures. Surely it leads to congestion in the system. Hence, in the first time of process, the whole system has to be done promptly. It helps to minimize the system to provide the feedback service.

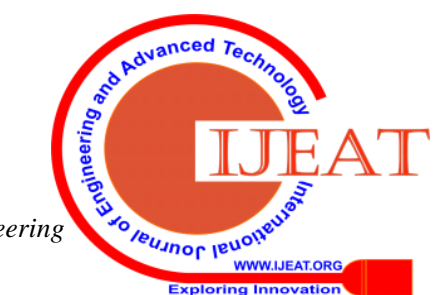


From table 1 obviously if the alternative of taking stage 3 administrations expands, the idle time of the server for this specific stage gets diminishes. Consequently the other performance estimates gets amplified. From table 2, the outcomes are true to form that is if the probability of getting into feedback service expands, it prompts an expansion in all the line execution measures. Surely it prompts a blockage in the system. Hence, in the first run through of process, the entire framework must be done promptly. It limits the framework to give the feedback administration.

\section{CONCLUSION}

In the above examination, the procedure of non-destructive testing is very much researched by the method of Queuing process. Supplementary variable method is connected to tackle the Queuing issue characterized and all the comparing lining execution measures are top notch. Numerical outline and graphical examination obviously gives a thought regarding the lining procedure considered in destructive testing.

\section{REFERENCES}

1. A.G.More and V.M.Bogar, "Condition Assessment of Bridges by NDT methods", International Journal of Engineering Development and Research, Vol.5, No.2, pp.191-196, 2017.

2. Alexander Nana Kwesi Agyenim-Boateng, Edward Kumi Diawuo and Psalmiel Nana Nti Agyei, "Dye Penetrant Inspection Technique of Turbine Rotating Component", International Journal of Modern Trends in Engineering and Research, pp.171-177, 2015.

3. J.Helal, M.Sofi, and P.Mendis, "Non-Destructive Testing of Concrete: A Review of Methods", Electronic Journal of structural Engineering, Vol.14, No.1, 2015, pp.97-105.

4. Masanori Fujita and Tomoya Masuda, "Application of various NDT Methods for the Evaluation of Building Steel Structures for Reuse", Materials, Vol.7, 2014, pp.7130-7144.

5. N.P.Migoun and N.V.Delenkovskii, "Improvement of Penetrant Testing Methods", Journal of Engineering Physics and Thermophysics, Vol.82, No.4, 2009, pp.737-744

6. K.C. Madan, and A. Baklizi, "An M/G/1 queue with additional second stage service and optional re service", International journal of information and Management sciences,Vol.16, No.2, pp.1-16,2002.

7. K.C. Madan, "On a single server queue with two stage heterogeneous service ad deterministic server vacations", The Egyptian Statistical journal, Vol.44, No.1, pp.39-55, 2000

8. S. Maragathasundari and S. Srinivasan, "Analysis of M/G/1 feedback queue with three stages and multiple server vacation", Applied mathematical sciences, Vol.6, No.125, pp.6221-6240, 2012.

9. S. Maragathasundari and S. Srinivasan, "A Non-Markovian Multistage Batch arrival queue with breakdown and reneging", Mathematical problems in engineering, Volume 2014/16 pages/ Article ID 519579/ http: // dx. Doi. Org / 10.1155/2014/ 519579, 2015.

10. F. A. Maraghi, K. C. Madan and K. Darby-Dowman , "Batch Arrival vacation queue with second optional service and Random Breakdowns", International Journal of Statistical Theory and Practice, Vol.4, No.1, pp.137-153, 2010.

11. N. Parida, "Non-destructive Testing and Evaluation of Cast materials", Materials characterization Techniques-Principles and Applications, pp.177-193, 1999.

12. D. A. Oke, G. F. Oladiran and S. B. Raheem, "Correlation between Destructive Compressive Testing (DT) and Non- Destructive Testing", International Journal of Engineering Research and Science, Vol.3, No.4, 2017, pp.27-30.

13. Paul Kah, Belinga Mvola , Jukka Martikainen and Raimo Suoranta "Real Time Non-Destructive Testing Methods of Welding", Advanced Materials Research ,Vol.93,2014,pp.109-116.

14. Q.Y.Lu and C.H. Wong, "Applications of Non-Destructive Testing Techniques for Post-Process Control of Additively Manufactured Parts", Virtual and Physical Prototyping, Vol.12, No4, 2017, pp.301-321.

15. Yu.A.Glazkov, "Evaluation of Material Quality for Liquid Penetrant Inspection Based on the Visibility of the Indicator Patterns of Flaws",
Russian Journal of Non-Destructive Testing, Vol.48, No.4, 2012, pp.208-217.

\section{AUTHORS PROFILE}

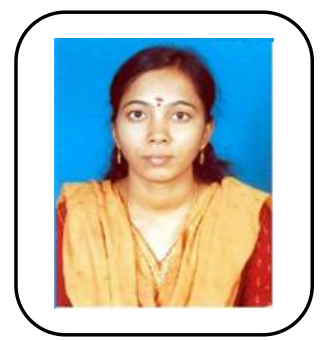

S. Radha, pursued her B.Sc degree from Manonmaniam Sundaranar University, Tirunelveli in 2006. She got her B.Ed. degree from V.P.M.M. College of Education, Srivilliputur in 2007 and her M.Sc. degree from Annamalai University, Chidambaram in 2009. She obtained her M.Ed. Degree from St. Justin's College of Education, Madurai in 2010. She has 4 years of teaching experience. Now, she is doing research in the area of Queuing theory, Kalasalingam Academy of Research And Education, Anand Nagar, Tamilnadu, Krishnankovil-626126, India.

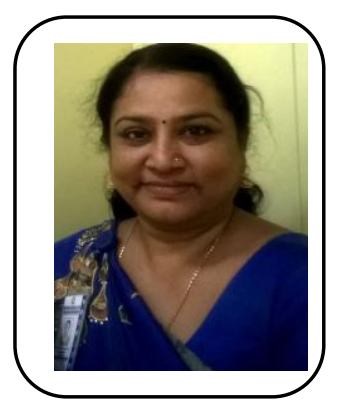

Dr. S. Maragatha Sundari, acquired her B.Ed. degree from V.O.C Teachers College, Tuticorin in 1993. She got her M.Sc. in Mathematics from Manonmaniam Sundaranar University; Tirunelveli in 1995 and her M.Phil. degree from Madurai Kamaraj University, Madurai in 2003. She did her Ph.D. in Sathyabama University, Chennai, India. She has more than 17 years of instructing knowledge. She has distributed more than 50 inquire about papers in national and international journals. She has displayed and distributed papers at national and international conferences. She is right now filling in as an Associate Professor in the Department of Mathematics in Kalasalingam Academy of Research And Education, Anand Nagar, Tamilnadu, Krishnankovil-626126, India. In addition, she is doing her research guidance for five research scholars.

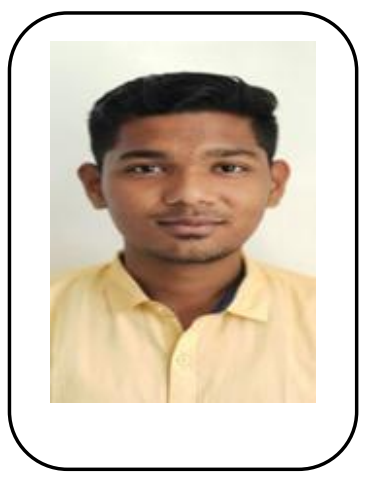

S. Kishore Eswar, pursuing his B.Tech Mechanical Engineering in KARE, Krishnankovil. He is a topper of his department and acts as a joint secretary of his department. He has participated in many science competitions and banged many prizes. Now, he is doing research applications in the area of Queuing theory. $\mathrm{He}$ is undergoing industrial implant training in TVS brake linings, Madurai.

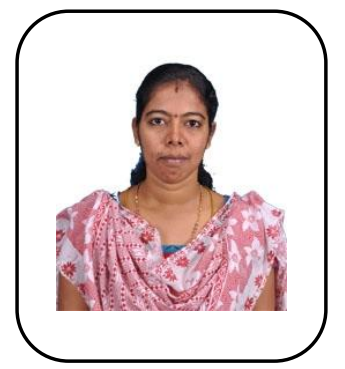

Sowmiyah.S, is currently working as a Assistant Professor in the Department of Mathematics, Velammal Institute of Technology, Chennai from 2011. She holds her Bachelor of Science degree in Mathematics from Seethalakhmi Ramaswami college, Tiruchirapalli. She completed Master of science with General Proficiency Award from Government college for women ,Kumbakonam. She completed Master of philosophy in Alagappa University. She have cleared SET-2016 conducted y Mother Theresa Women's University. She has twelve years of teaching experience in private engineering colleges and a life member of AICTE. She has published two journals along with Dr.S.Maragathasundari. 